\title{
Treatment of Lumpy Skin Disease
}

\author{
Shawn Babiuk
}

Unfortunately there are no specific antiviral drugs available for the treatment of lumpy skin disease. The only treatment available is supportive care of cattle. This can include treatment of skin lesions using wound care sprays and the use of antibiotics to prevent secondary skin infections and pneumonia. Anti-inflammatory painkillers can be used to keep up the appetite of affected animals. Intravenous fluid administration may be of benefit; however this may not be practical in the field. The lack of treatment options for lumpy skin disease virus emphasizes the need of using effective vaccination for preventing disease. 